\title{
Wind Turbine Aerodynamics from an Aerospace Perspective
}

\author{
A. van Garrel ${ }^{1}$, S. ten Pas $^{2}$, and C.H. Venner ${ }^{3}$ \\ University of Twente, Enschede, 7500AE, The Netherlands \\ and \\ J. van Muijden ${ }^{4}$ \\ Netherlands Aerospace Centre (NLR), Amsterdam, 1059CM, The Netherlands
}

\begin{abstract}
The current challenges in wind turbine aerodynamics simulations share a number of similarities with the challenges that the aerospace industry has faced in the past. Some of the current challenges in the aerospace aerodynamics community are also relevant for today's wind turbine aerodynamics community and vice versa. This paper sketches these similarities in broad strokes and points out the possibilities to revive solutions from the aerospace aerodynamics community from the 1960's onward that, with some modifications, can be applied to wind turbine aerodynamics problems. Opportunities are indicated where both the wind turbine and the aerospace aerodynamics communities can benefit from collaborative research.
\end{abstract}

$\begin{array}{ll}B E M & =\text { Blade-Element-Momentum } \\ C F D & =\text { Computational Fluid Dynamics } \\ C p & =\text { Power coefficient } \\ C O E & =\text { Cost of Energy } \\ I B L & =\text { Integral Boundary Layer } \\ L E S & =\text { Large Eddy Simulation } \\ \text { RaNS } & =\text { Reynolds-averaged Navier Stokes } \\ V I I & =\text { Viscous-Inviscid Interaction } \\ \lambda & =\text { Tip speed ratio (rotor tip speed/wind speed) }\end{array}$

\section{Introduction and Historical Overview}

$\mathrm{T}$ HE utilization of aerodynamic forces for industrial activities in the Netherlands took flight in the late 1500's by the invention of the wind powered sawmill. Now wooden planks could be made 30 times faster than previously and this enabled the construction of a massive fleet of ships. Wind energy thus formed part of the basis of the "Dutch Golden Age" that spanned roughly from 1600 to 1700 in which the Dutch Republic was the foremost maritime and economic power in the world. At the time the steam engine was introduced in the Netherlands (around 1850), an estimated number of 10,000 wind mills were in use. During the industrial revolution the use of coal as source of energy led to a gradual decline of the use of wind as a means to do work. This decline in wind energy utilization was finalized with the discovery of cheap and abundant oil.

It was the quest for flying machines that drove the interest in the further development of a theory for aerodynamics around the start of the twentieth century. Some of the notable names that also greatly contributed to wind turbine and propeller aerodynamics are Joukowsky in Russia, Prandtl and Betz ${ }^{1}$ in Germany, and Lancaster in Great-Britain.

\footnotetext{
${ }^{1}$ Research Scientist, Faculty of Engineering Technology, Engineering Fluid Dynamics, P.O. Box 217, a.vangarrel@utwente.nl.

${ }^{2}$ MSc Student, Faculty of Engineering Technology, Engineering Fluid Dynamics, P.O. Box 217, sebastiaantenpas@gmail.com.

${ }^{3}$ Professor, Faculty of Engineering Technology, Engineering Fluid Dynamics, P.O. Box 217, c.h.venner@utwente.nl.

${ }^{4}$ Reseach Scientist, Aerospace Vehicles, Flight Physics \& Loads, P.O. Box 90502, Jaap.van.Muijden@nlr.nl.
}

American Institute of Aeronautics and Astronautics 
It was Betz ${ }^{2}$ in 1926 in the introduction of a paper dedicated to wind turbine aerodynamics who strongly recommended that more research efforts should be directed towards wind energy. The coal shortage mentioned by Betz in his recommendation for wind energy was due to the in-kind reparations that Germany had to submit to after their defeat in WW-I. This bears some similarity with the current situation in which there is a growing hunger for energy while conventional resources are past their peak and climate change consequences are clear. The aerodynamic theories developed in that era by the research groups in Russia, Germany, and England are still in use today by wind turbine designers as blade-element-momentum (BEM) methods. An overview of the ideas behind BEM methods can be found in the reference work by de Vries ${ }^{3}$ or the recent book by Sørensen ${ }^{4}$, and a discussion of the work by Joukowsky can be found in Ref. 5 and Ref. 6.

More practically oriented scientist and inventors that demonstrated the use of aerodynamic forces for manned flight are, amongst others, Otto Lilienthal, Alberto Santos-Dumont, and Wilbur and Orville Wright. These pioneers captured the imagination of the world with their demonstrations of flying airplanes and spurred the drive towards understanding the physics of aerodynamic flows.

The development of the theory for aerodynamic flows was greatly motivated by the wars of the twentieth century: WW-I, WW-II, and the cold war. After WW-II, the general availability of the digital computer in 1957 heralded the birth of computational fluid dynamics (CFD) without the need for classrooms full of human computers as shown in Figure 1 and described by Grier in Ref. 7. It was the start of research into areas that were unimaginable before and the beginning of an explosion of algorithms, theories, mathematical models, approximations, books, articles, and computer programs for the simulation of the behavior of fluids under various circumstances.

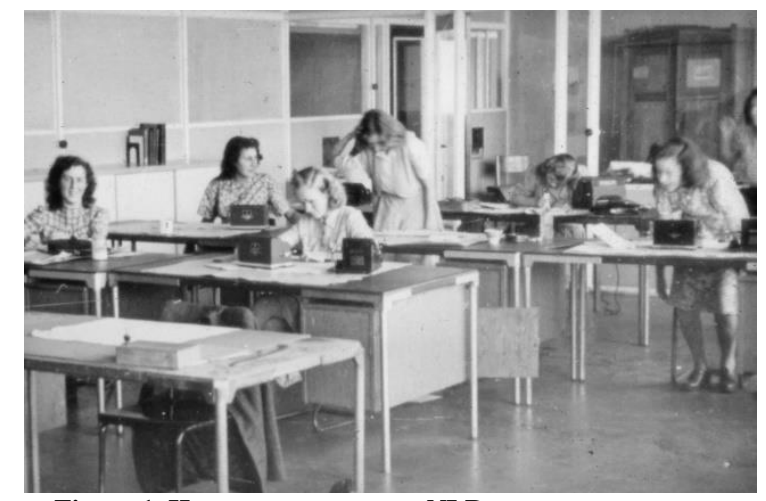

Figure 1 Human computers at NLR. Courtesy of H.Tijdeman.

A renewed interest in wind energy was sparked due to the oil crisis of 1973 when Middle Eastern countries increased oil prices by $70 \%$ and reduced oil output every month. In many countries national programs were initiated and tasked with the solution to this "energy crisis", where wind energy was an important ingredient. For example, in the USA the precursor to the Department of Energy tasked NASA with research into utility-scale wind turbines. In cooperation with large companies like Boeing, Lockheed, and General Electric from the aerospace field, a series of prototype wind turbines were developed ranging from the 38-meter rotor diameter MOD-0 turbine of $100 \mathrm{~kW}$ rated power in 1975, to the 97.5-meter rotor diameter MOD-5B turbine of $3.2 \mathrm{MW}$ rated power in 1987. It was soon discovered that the knowledge gained in the aerospace industry on aerodynamics and structures was not one to one transferable to the realm of the highly unsteady characteristics prevalent in wind turbine applications.

The same oil crisis was also the reason for an increase in research into improving the aerodynamic efficiency of airplanes. Among the topics for this research were the development of computer models for laminar-turbulent transition, alternative high aspect ratio wing configurations, high bypass-ratio engines, reducing (shock) wave drag, and counter-rotating propfan aircraft configurations, to name a few.

The resolution of the oil crisis and a return to low cost oil from the Arab countries made the argument for investing in renewable energy very difficult. The result was an adverse business climate for wind turbine manufacturers and a discouragement for the installation of wind turbines. The same fate was reserved for the research in the aerospace industry on increasing aerodynamic efficiency where focus returned towards bigger and faster airplanes with larger range.

Nowadays there is again a confluence of challenges facing both the aerospace industry and the wind turbine industry. The switch from fossil fuels to renewable energy sources is motivated by the effects of climate change and the reduced availability of easily extractable cheap oil. This also necessitates a reduction in greenhouse gas emissions and an overall increase in efficiency in the aircraft performance and research in alternative energy carriers. In this article we will focus on aerodynamics related issues where the two industries share similar problems and cooperative research would benefit both. The discussion gravitates towards numerical simulation methods. When applicable we will also mention the differences in perspective the two industries have. 


\section{Comparison of Technologies and Challenges}

\section{A. Wind Turbine Aerodynamics and Aeroelasticity}

The quest for a lower Cost of Energy (CoE) by wind energy conversion systems is the main driver behind the increasingly larger wind turbines. The wind power generated by a wind turbine is linearly dependent on the rotor area and thus scales quadratically with the blade length. Currently the largest wind turbine rotor blade is produced by LM Wind Power and has a length of 88.4 meters, i.e. a rotor diameter of at least 176.8 meters. In comparison, the largest aircraft in the world, the Airbus A380, has a wing span of just under 80 meters. Figure 2 compares the A380 and a wind turbine from 2012 with the formerly largest rotor blade by Siemens with a length of 75 meters.

The increased wind turbine dimensions give rise to increasing investment costs and related concerns regarding risk mitigation. An increase in size also leads to relatively flexible structures that are more susceptible to unsteady load occurrences. Such fluctuating loading is inherent in unsteady wind turbine aerodynamics and caused for example by variations in wind speed and direction in space and time, irregular rotational speed, blade pitch actions, rotor yaw misalignment, blade deformations, the dynamic character of the wake downstream of the rotor, and wake - wind turbine interactions (see Figure 3), to name a few.

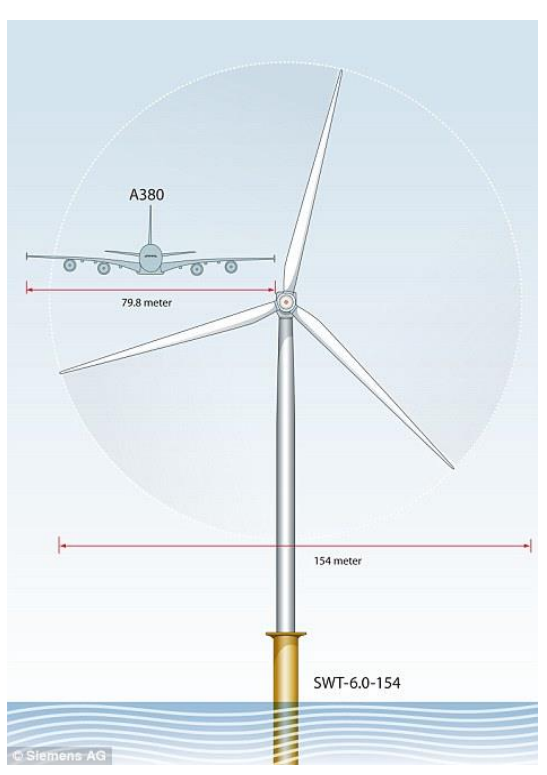

Figure 2 Airbus A380 versus Siemens 6 MW wind turbine.

Such large-scale wind turbines will be deployed in future wind farms as depicted in Figure 3. The range of scales in space and time that govern the aerodynamic properties of such wind farms ranges from millimeters and microseconds for the rotor blade boundary layer to kilometers and minutes for the effects of the wind turbine wake. When the earth boundary layer and the weather patterns are also considered the range of scales increases further. The large range of scales makes it infeasible to use the highest fidelity numerical simulation methods based on the NavierStokes equations for the analysis of full wind farm configurations. For these cases approximations are introduced for

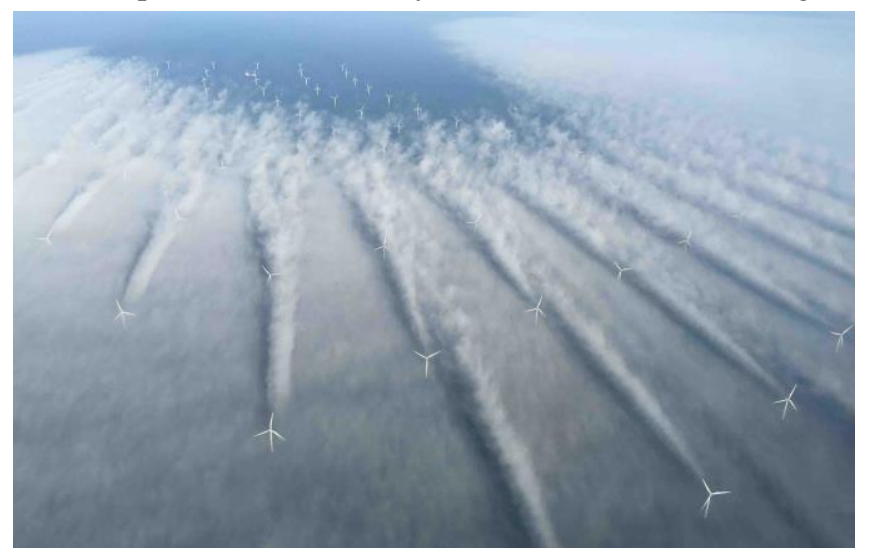

Figure 3 Offshore wind farm. the representation of the flow physics and for the representation of the geometry. A reasonable approach, especially in case of wind farm optimization, is for example to use the parabolized Navier-Stokes equations with a model for the turbulence and to represent the wind turbine by an actuator disk or by actuator lines ${ }^{8,9,10}$. Wind farms are inherently operating in unsteady flow conditions. The operation in upstream wind turbine wakes can aggravate the unsteady character. Floating offshore wind turbines introduce another source for the dynamic behavior and introduce the problem of describing sea wave conditions. Time-averaged models for wind farm simulations can therefore not be used reliably for the time-local analysis of individual turbines in the wind farm.

For the numerical simulation of the aerodynamic characteristics of a single wind turbine near design conditions the state-of-the-art methods based on the Navier-Stokes equations demonstrate a good agreement (see ten Pas ${ }^{11}$ ) with experiments conducted in a wind tunnel (see Boorsma ${ }^{12}$ ). Even in these cases there are some discrepancies between numerical and experimental results. One of the topics that can be improved upon is the prediction of laminar-turbulent boundary layer transition. It must be noted that there is detailed aerodynamic data available for only a limited number of scaled wind turbines ${ }^{12,13}$ that were submitted, under controlled conditions, to extensive wind tunnel tests. In these tests the model wind turbine rotor blade deformations were negligible and not representative for the full-scale situation. Such tests with structurally representative rotor blade designs are highly recommended. 
For the analysis of solitary wind turbines operating in unsteady conditions the use of high fidelity simulation methods based on the Navier-Stokes equations in an engineering environment is limited to a few specific load cases, due to the associated computational costs. This is the reason that for the analysis of wind turbines for the whole spectrum of load cases during the certification process the classical Blade-Element-Momentum (BEM) theory from the days of Prandtl and Betz ${ }^{1}$ is still used to predict the aerodynamic loads. These classical BEM methods are nowadays extended with an increasing number of corrections ${ }^{4}$ that try to remedy the shortcomings introduced by the assumptions of the essentially one-dimensional theory for steady flows

The challenge for wind turbine applications is to ultimately integrate a mix of simulation methods for unsteady aerodynamics, structural dynamics, and control strategies for use in a wind turbine engineering environment with balanced problem turnaround times and simulation accuracy. The success of flow solvers based on the Navier-Stokes equations for aerospace applications, often using large computer systems (many cores), is unfortunately not one-toone transferable for day-to-day practical use in a wind turbine engineering environment due to the computational burden associated with the inherently unsteady flow conditions. There is however still a need for an aerodynamics simulation method with higher fidelity than the BEM methods currently in use in that mix of simulation methods while maintaining reasonable computational demands and short problem turnaround times on relatively small-scale computer systems.

A consequence of the increasing size of the wind turbine rotor blades is the increasing in-plane blade root bending moment due to gravitational forces acting on the blade. The increased loading on the trailing edge of the blades can cause problems in their structural integrity. To counter these problems airfoils with thick trailing edges have been designed (e.g. Ref. 14) that can simultaneously reduce the adverse pressure gradients on the suction side of thick airfoils and thereby improve the airfoil lift force. Such airfoils however have different aerodynamic characteristics than their sharp trailing edge counterparts. Near the blade root the increase in drag associated with the blunt trailing edge has a limited effect on the power performance of the rotor as the drag force vector has a large component in the rotor axial direction and a relatively small in-plane component contributing negatively to the aerodynamic torque of the rotor. Near the blade root the detrimental effect of the increased drag however can be compensated or even turned into a net positive effect by the increase in lift.
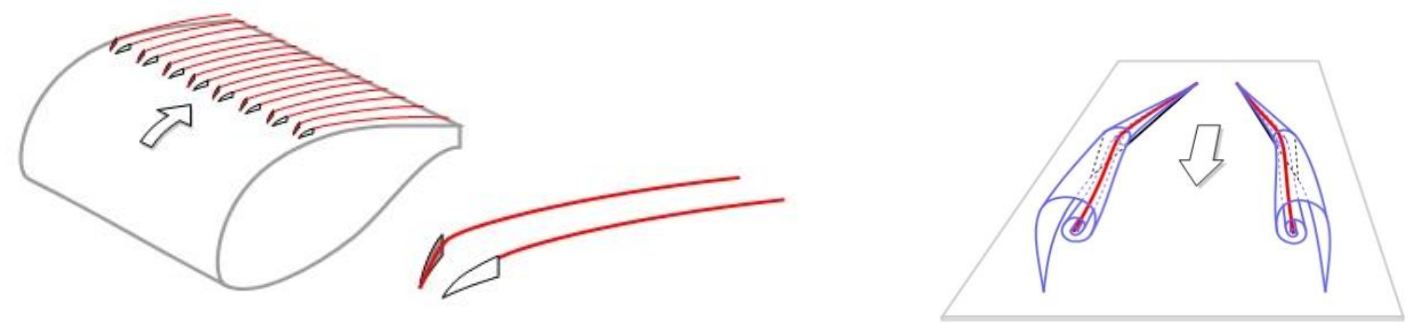

Figure 4 Thick trailing edge airfoils and vortex generators for wind turbine applications.

To increase rotor blade airfoil thickness and avoid large deformations out of the rotor plane, currently a focus is on vortex generators that distribute high energy flow from outside the rotor blade boundary layer to the low energy flow regions very close to the blade surface (see Figure 4). The numerical simulation of the strong interaction between the confluent boundary layers from the airfoil and the vortex generator is currently pursued in research environments but still with limited success. The goal is to be able to design new thick airfoils that have vortex generators incorporated from the outset.

Wind turbine design simulations that involve aeroelasticity are characterized by the large number of simulations that are needed for certification and the high degree of coupling with controller strategies for pitch, yaw, brakes, and the generator. The aerodynamic and structural dynamic behavior of a wind turbine in the time domain is therefore often simulated by a combination of simple Blade-Element-Momentum methods for the prediction of the aerodynamic forces, and beam models of varying fidelity for the (multi-body) dynamic characteristics of the blades and tower. The cross-sectional structural properties used in these simulations come from higher fidelity FEM models that can for example account for the detailed composites material layup (individual plies) of the thick shell rotor blade elements.

American Institute of Aeronautics and Astronautics 
An essential element in wind turbine aeroelasticity simulations is the stochastic character of the onset wind velocity that should be accounted for by synthetic turbulence generator models as for example developed by Veers ${ }^{15}$, Winkelaar ${ }^{16}$, Kelley ${ }^{17}$, or Mann ${ }^{18}$.

Engineering models for the effect of soil-foundation interaction, and water wave loading in case of offshore wind turbines, are also to be taken into account in wind turbine dynamics simulations. Note that also high fidelity methods are currently being developed for wind turbine aeroelasticity simulations, see for example the work by Hsu and Bazilevs ${ }^{19}$. Research on LIDAR based forward-looking scanning techniques for the incoming wind flow field offers the promise of preemptive control actions. Floating offshore wind turbines introduce new challenges for aeroelastic simulations and control.

\section{B. Aerospace Aerodynamics and Aeroelasticity}

The current hot topics in aerospace aerodynamics are largely defined by political targets on emissions and noise reductions to be achieved by 2050, both in the USA and in Europe. As a result, the investigations on how to reduce the noise and carbon footprint of air transportation can be characterized by evolutionary developments on one hand and by revolutionary developments on the other hand. The evolutionary developments focus on the optimization of existing ideas and technologies. Drag reduction and cruise optimization have gained renewed interest, leading to studies on enhanced laminar flow regions on transport aircraft as well as increasing fuel efficiency of gas turbines. The revolutionary developments focus on new, efficient and more silent propulsion techniques like the contra-rotating open rotor, boundary layer ingestion gas turbines, alternative sources of energy (mostly electrical) and their impact on aerospace vehicle design and propulsion, less drag generating configurations (e.g. morphing structures in stead of flap deflections, high aspect ratio wings) and forms of on-board electricity generation and distribution. These concepts lead to numerous yet unsolved problems for which many solution ideas are being suggested. However, none of the proposed solutions is yet sufficiently mature to emerge as an undoubtedly viable stepping stone for future aerospace vehicles.

Current state-of-the-art in aerodynamic analysis and optimization for aerospace applications is largely based on Navier-Stokes CFD methods that are capable of capturing highly complex geometries, rotating frames, complex flow phenomena (laminar-turbulent transition, vortex flow, jets, compressibility, shock waves) and their associated noise generation. This does not necessarily imply that simpler methods are no longer used. The simpler methods (e.g. unsteady vortex-lattice methods, full potential methods in combination with boundary integral methods) have their own strong potential in preliminary design or detailed design, or for a quick assessment of a vast number of conditions, e.g. in aerodynamic loading over the flight envelope for certification purposes. However, for the detailed and accurate assessment of current fluid flow problems of interest, selecting one of the forms of viscous fluid flow modelling (RaNS, unsteady RaNS, Detached Eddy Simulation, Large Eddy Simulation) has become standard. The choice of viscous flow modelling depends on the application at hand.

The verification and validation of computational methods in aerodynamics are indispensable and performed either by comparison with experimental data from wind tunnel tests or flight tests. This is currently more of an issue for the wind turbine industry for two reasons: good wind tunnel experiments on scaled wind turbines are scarce and field experiments suffer largely from the unknown unsteady turbulent flow conditions under which the full-scale wind turbine operates.

The push towards lowering $\mathrm{CO}_{2}$ emissions shifts the focus again towards increased aerodynamic efficiency and lower aircraft weight. To increase the aircraft lift-to-drag ratio higher aspect ratio aircraft wings and large areas of (passive or active) laminar flow are key. A higher wing flexibility and larger structural deformations are to be expected and consequently the accurate simulation of aeroelastic conditions will gain importance. For the simulation of laminarturbulent flow transition research is ongoing at NLR. For an improved propulsion efficiency engine, boundary layer ingestion and/or unducted fan configurations are topics currently under investigation. Electric propulsion is also considered, although a break-through technology is required to solve the energy storage problem. High lift, multielement configurations for take-off and landing configurations are also subject of research to combat $\mathrm{CO}_{2}$ emissions. The overlap with wind turbine technology is mainly found in solving problems in the area of aeroelasticity and with a better prediction of laminar-turbulent transition. Safety is one of the areas where the differences between the wind turbine industry and the aerospace industry are pronounced and translate into differences in manufacturing costs.

American Institute of Aeronautics and Astronautics 
At NLR, the validation of state-of-the-art aerospace CFD-technology for a specific wind turbine experiment (MEXICO: (Model EXperiments In COntrolled conditions) has been performed within a master thesis study (Ten $\mathrm{Pas}^{11}$ ) aiming at investigating the applicability of existing technology to a practical and realistic wind turbine flow problem. The results are very promising. The application of a multi-block structured compressible flow solver (ENSOLV, NLR in-house developed, part of the ENFLOW CFD-system) to the flow about the MEXICO rotor as measured in the DNW LLF wind tunnel has shown that high-quality predictions can be obtained using state-of-theart high-precision aerospace computational technology on the basis of steady Reynolds-averaged Navier-Stokes equations (other available, more advanced options include unsteady and hybrid RaNS-LES flow simulations). The configuration used in this validation study is shown in Figure 5 as tested in the open test section of the wind tunnel (left) and as modelled for CFD (right).
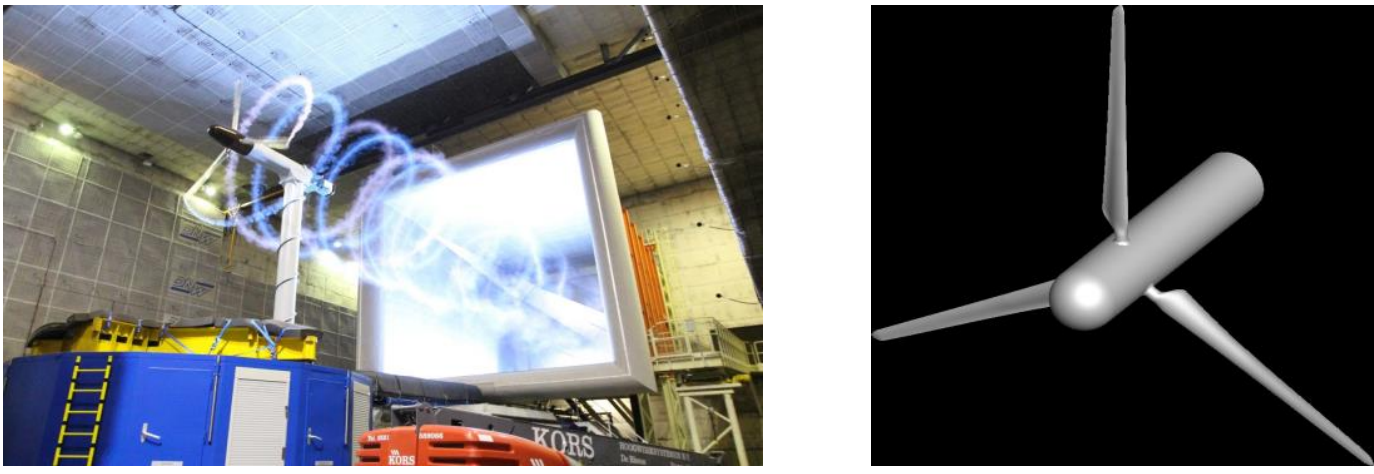

Figure 5 MEXICO model wind turbine.

Figure 6 gives the different types of airfoils used to define the rotor blade of the MEXICO wind turbine, together with the transfer zones between each pair of airfoils. Each airfoil is connected to specific flow characteristics, and is not necessarily the best choice for application in a realistic wind turbine rotor. Nevertheless, this variation in airfoils

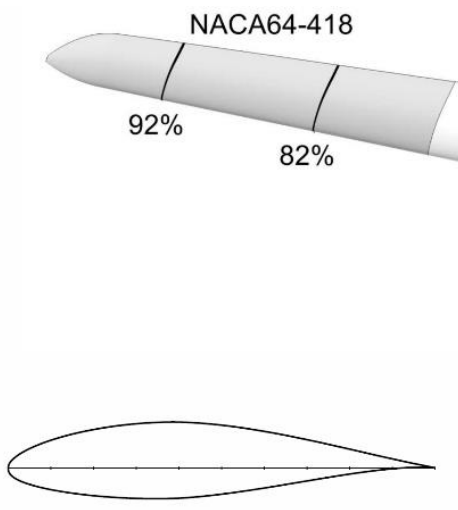

(a) NACA64-418 airfoil.

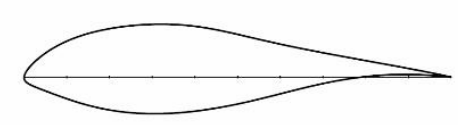

(b) Risø A2-21 airfoil.
DU91-W2-250
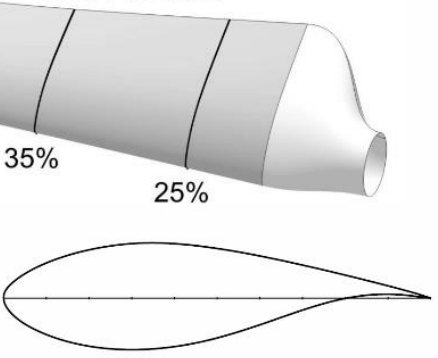

(c) DU91-W2-250 airfoil.

Figure 6 MEXICO model wind turbine rotor blade and defining blade cross-sections.

poses a demanding and interesting test case for validation of the flow solver.

A comparison of results from computations and experiment are shown in Figure 7 for the design case of zero degrees yaw, blade pitch angle of $-2.3^{\circ}$, a wind speed of $14.7 \mathrm{~m} / \mathrm{s}$, and a tip speed ratio of 6.8 . The error bars on the experimental results as shown in the plots are indicative for the expected uncertainty in measured pressure due to the rotation. The accuracy of the selected pressure transducers is well balanced to the prevailing dynamic pressure at the rotating tip of the turbine, whereas the accuracy of experimental pressure data decreases towards the hub. In addition to the results obtained by the ENSOLV numerical solution method the results obtained by a fast multilevel panel method $^{20,21}$ that models inviscid, incompressible flow are also shown. 


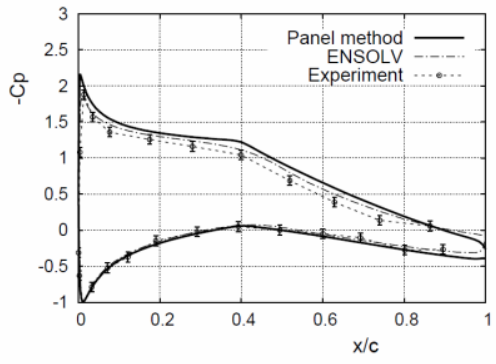

(a) $92 \%$ Rotor radius, NACA64-418.

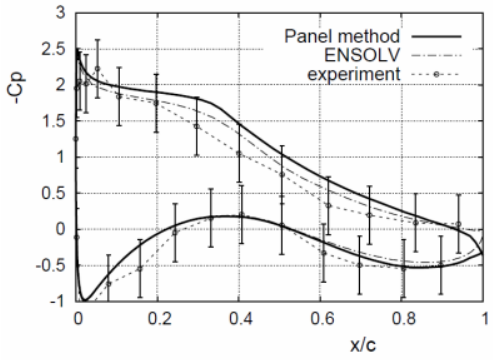

(d) $35 \%$ Rotor radius, DU91-W2-250.

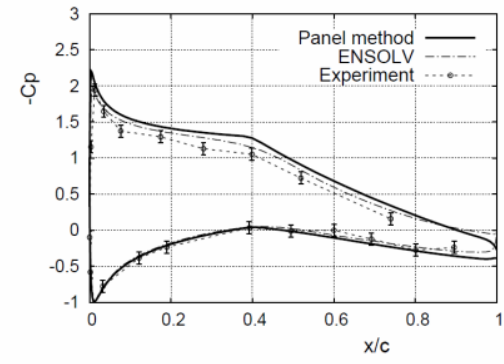

(b) $82 \%$ Rotor radius, NACA64-418

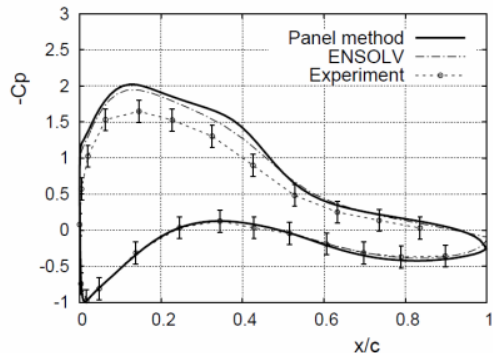

(c) $60 \%$ Rotor radius, Risø A2-21.

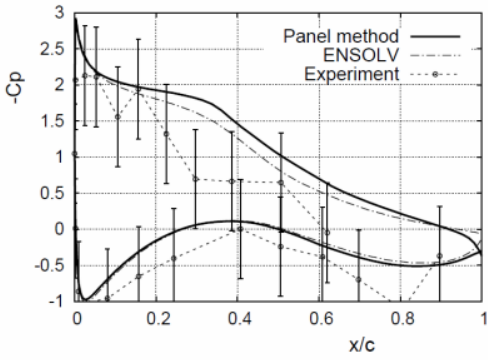

(e) $25 \%$ Rotor radius, DU91-W2-250.

Figure 7 MEXICO rotor blade surface pressure distributions at design conditions.

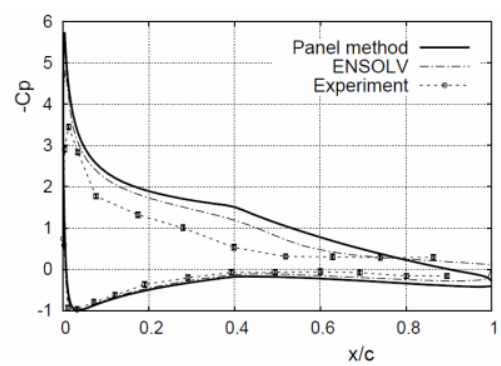

(a) $92 \%$ Rotor radius, NACA64-418.

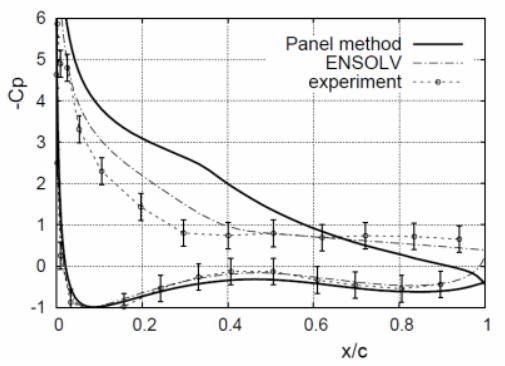

(d) $35 \%$ Rotor radius, DU91-W2-250.

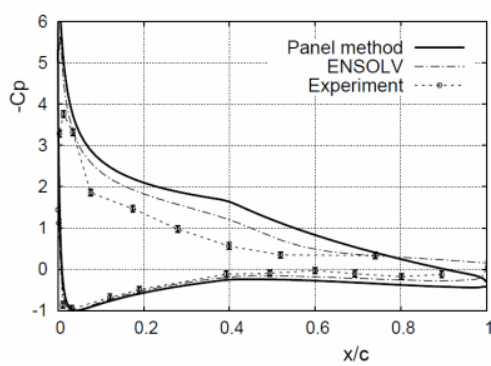

(b) $82 \%$ Rotor radius, NACA64-418

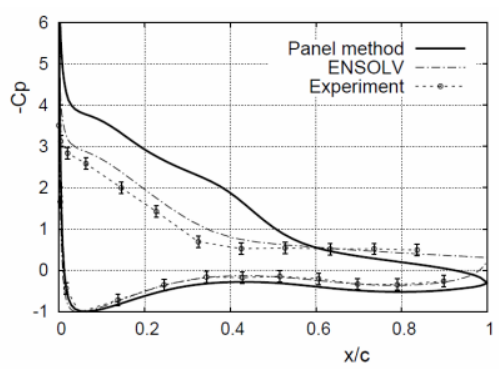

(c) $60 \%$ Rotor radius, Risø A2-21.

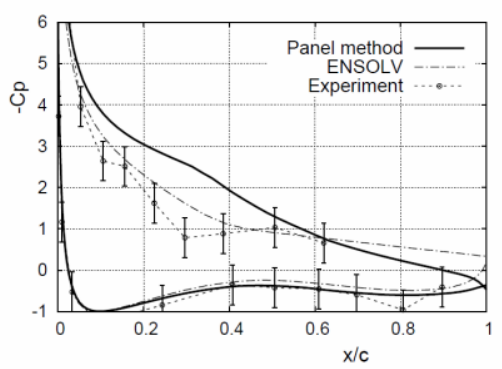

(e) $25 \%$ Rotor radius, DU91-W2-250.

Figure 8 MEXICO rotor blade surface pressure distributions at separated flow conditions. 
Other cases computed with the ENSOLV numerical solution method and compared with experimental data consist of a turbulent wake state (wind speed of $10 \mathrm{~m} / \mathrm{s}$, tip speed ratio of 10), a separated flow case (wind speed of $24.12 \mathrm{~m} / \mathrm{s}$, tip speed ratio of 4.2) and a standstill condition (wind speed of $29.92 \mathrm{~m} / \mathrm{s}$, tip speed ratio 0), the latter only being instructive for the assessment of aerodynamic loads predictions on the rotor in vane position. Figure 8 shows the comparison for the separated flow case. Here the panel method is inadequate as it does not capture the strong influence that viscosity has on the flow field.

The assessment of the power coefficient of the wind turbine is of utmost importance for its performance and expected economic viability. The power curve as obtained for the MEXICO wind turbine from the ENSOLV CFDresults is shown below for a rotor with a rotational speed of $425.1 \mathrm{rpm}$. A maximum power coefficient of about 0.435 is observed at a tip speed ratio of 7.5.
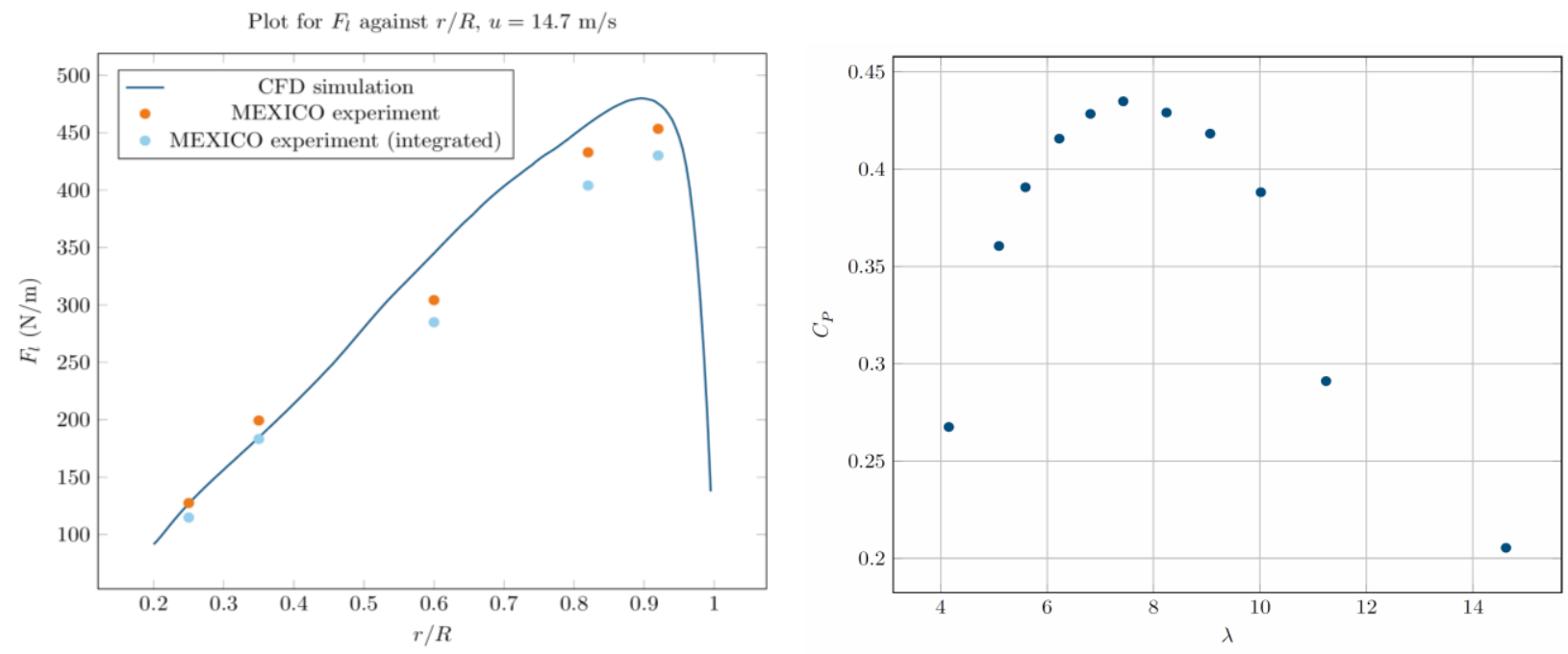

Figure 9 MEXICO rotor tangential force distribution at design conditions and power curve for varying tip speed ratios.

The favorable results for the multilevel panel method shown in Figure 7 encouraged us recently (see Prieto ${ }^{22}$ ) to validate the multilevel panel method for unsteady flow cases. Figure 10 shows some preliminary results for the rotor at $45^{\circ}$ yaw and design flow conditions. The surface pressure distributions for the blade at $240^{\circ}$ azimuth angle (8 o'clock position, advancing blade) at $60 \%, 82 \%$, and $92 \%$ blade radius are shown. The differences between the pressure distributions from the experiment and from the numerical simulation are small for the blade position shown. All other blade positions considered in the study show a similar difference. For flow conditions with flow separation occurring at the blade the comparison was less favorable: the effects of the boundary layer on the pressure distribution have to be accounted for in these cases.

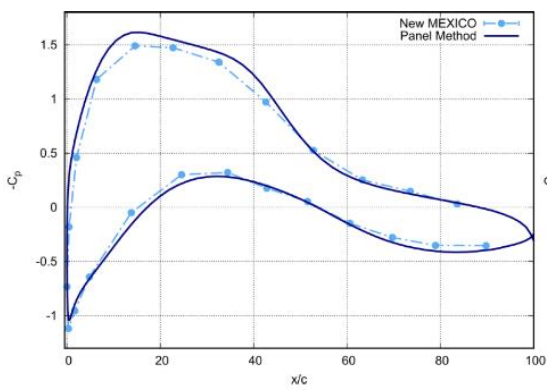

$60 \%$ Rotor radius, Ris $\varnothing \mathrm{A} 2-21$

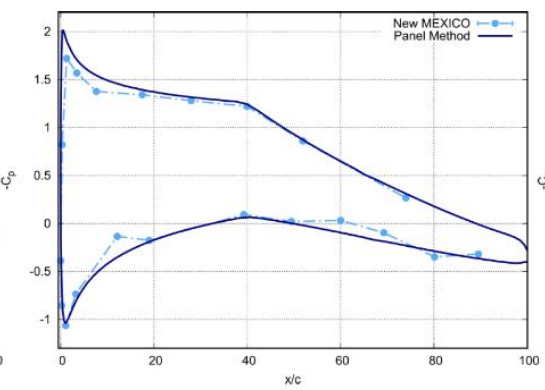

$82 \%$ Rotor radius, NACA64-418

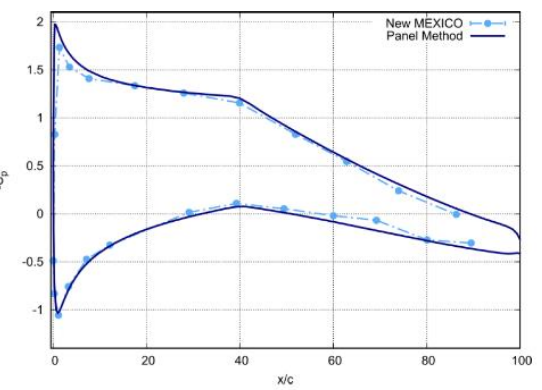

$92 \%$ Rotor radius, NACA64-418

Figure 10 MEXICO rotor surface pressure distributions. Rotor at $45^{\circ}$ yaw angle. Blade position at $240^{\circ}$ azimuth angle.

For aerospace aeroelasticity, the current state-of-the-art is heavily linked to the advances made in aerodynamics modelling and structural modelling. For the structure, either coarse or fine finite element models can be applied, depending on the focus of research (flutter and loads versus stresses and fatigue life). These FEM-models are most of

American Institute of Aeronautics and Astronautics 
the time linear in nature, meaning that applying loads to the structure leads to deformations of the structure in the direction of the loads. Nonlinear effects between loads and deformations (buckling, permanent deformations etc.) are usually ignored, assuming normal operating conditions. The deformations of the structure are derived from the prevailing inertia and aerodynamic loads, and implemented in a loosely coupled approach. The loosely coupled approach iterates between aerodynamic and structural computations, each having an optimized mesh resolution. Communication of data between the aerodynamic and structural meshes in this approach requires interpolation. The loads on the structural mesh are transformed into deformations. The deformations are transformed to the aerodynamic mesh. The shape of the configuration is deformed during iterations between aerodynamic and structural calculations. Validation of structural models is necessary for flutter applications (mode shape matching/checking between FEM and actual configuration through ground vibration test) and for stress applications (deformation verifications).

\section{Wind Turbine Aerodynamics - Reviving Aerospace Aerodynamics Solutions}

Wind turbine aerodynamics simulation tools that can predict the effects of unsteady flow on the loading on the wind turbine rotor blades with sufficient accuracy are key to more economic wind turbine designs in the near future. Despite the advances in numerical methods based on the Navier-Stokes equations the computational efforts for the simulation of the unsteady flow around wind turbines are too demanding to be applicable in an engineering environment. The challenge for wind turbine aerodynamics simulation methods is therefore to obtain higher fidelity solutions than the methods based on Blade-Element-Momentum theory while keeping the computational burden limited.

A possible solution to this challenge is the Viscous-Inviscid Interaction (VII) technology that was developed in the 1970's where inviscid flow methods were coupled in strong interaction with a method solving the boundary layer equations in order to account for the effects of viscosity, specifically in the boundary layers. An overview of the aspects involved in such an approach has been given by Lock and Williams ${ }^{23}$. As mentioned earlier, this research was almost completely abandoned once practically applicable RaNS based computational methods started to appear. Drela $^{24}$ was one of the first to implement a computational method that coupled a panel method for the inviscid external flow with an Integral Boundary Layer (IBL) method successfully for two-dimensional airfoils. His XFOIL computer program is nowadays open source, freely available ${ }^{25}$, and the de-facto standard in the wind turbine industry.

For wind turbine and helicopter rotor blades the XFOIL program for two-dimensional flows was extended with a boundary layer approximation for the radial flow in the rotating airfoil boundary layer. The resulting RFOIL code was created in a collaborative project of ECN, NLR, and Delft University of Technology by Snel, Houwink, Bosschers ${ }^{26}$, and Van Rooij ${ }^{27}$. Later improvements were applied to the RFOIL program for a more accurate prediction of drag and lift by Özdemir and co-workers ${ }^{28,29,30}$. A similar approach that models a quasi-3D boundary layer flow, but now coupled with a fully 3D panel method, has been pursued by Ramos-García ${ }^{31}$ in cooperation with Sørensen and Shen ${ }^{32}$ in Denmark.

At NLR in the Netherlands, a successful implementation of a method solving the 3D full-potential equations in strong interaction with a finite difference method for the 3D integral boundary layer (IBL) equations found its way into the MATRICS-V code by Van der Wees and Van Muijden ${ }^{33}$. Due to its ease of use and the fast problem turnaround times this computational method was part of the core aerodynamic design tools in the engineering design environment at Fokker Aircraft for the simulation of steady transonic flows about simple wing-body configurations. The quasisimultaneous viscous-inviscid interaction theory that laid the foundation for this success was developed at NLR by Veldman $^{34}$.

At ECN a project was initiated in 2003 with the objective to create RotorFlow ${ }^{35,36}$, an unsteady and fully $3 \mathrm{D}$ equivalent of the successful XFOIL/RFOIL codes. In parallel to the development of a panel method for threedimensional unsteady flows ${ }^{36}$, Özdemir and co-workers performed various studies to solve the unsteady form of the integral boundary layer equations employing a high-order discontinuous Galerkin method with a non-conservative flux scheme ${ }^{37,38}$ together with the quasi-simultaneous interaction method of Veldman ${ }^{34,39}$. In a recent publication Özdemir et al. demonstrated a proof-of-concept of this method ${ }^{40}$. A fully 3D approach for steady flows is currently also investigated by Drela and fellow researchers ${ }^{41,42}$.

For wind turbine applications the operational speeds are in the low subsonic regime and this VII technology could be used to couple an incompressible inviscid solution provided by a so-called panel method with an integral boundary

American Institute of Aeronautics and Astronautics 
layer method to account for the effects of viscosity. An accurate prediction of laminar-turbulent transition in the boundary layer would be part of such a numerical simulation method.

Recent progress ${ }^{20,21}$ in reducing the computational burden of the classical panel method for wind turbine applications makes this approach an attractive proposition. Figure 11 shows an example of achieved reductions in

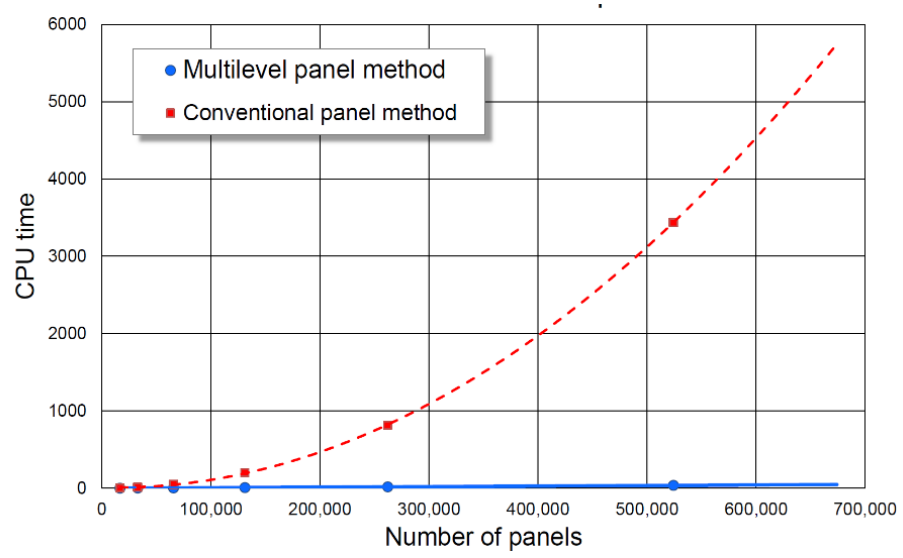

Figure 11 Fast multilevel panel method CPU time compared to the classical panel method.

integral evaluation time for the newly developed multilevel panel method. Both elements of the proposed VII solution, i.e. the panel method and the integral boundary layer method with transition prediction, only require the surface of the configuration to be discretized. This facilitates short problem turnaround times in a wind turbine engineering design environment as the cumbersome generation of a grid in the volume surrounding the configuration is avoided. Of course, such numerical simulation method would also find its way into the analysis of low speed subsonic flow regime problems in the aerospace industry.

At NLR another interesting development in the early 1980's has been the so-called field panel method ${ }^{43,44}$ that was based on the full potential equation for isentropic flows. Its advantage was that only the part of the flow with nonnegligible compressibility required a volume grid. The project, however, was put on hold due to the impractical quadratic computational costs. Later the project was reconsidered after the fast multilevel algorithm by Brandt and Lubrecht ${ }^{45}$ was found to reduce this cost substantially. However, by that time the vast investments in numerical flow simulation methods based on the Euler equations, their successful application, and the prospect of extending the method to general viscous flows was the reason the project was abandoned for good. For wind turbine designs, where the trend is towards higher subsonic rotor tip speeds (currently $\sim 100 \mathrm{~m} / \mathrm{s}$ ), this approach could be revived while keeping the computational costs limited through the use of the fast multilevel panel method ${ }^{20,21}$.

\section{Opportunities for Collaborative Research}

There are multiple technology areas where both aerospace and wind energy can benefit from collaborative research. Including the mutual interests mentioned in the preceding sections some of the identified overlapping topics in aerospace and wind turbine technology are (in random order):

- Laminar-turbulent transition

- Separated flow assessment

- Aerodynamic loads predictions over the operational envelope

- Composite structure deformation predictions in relation to adequate FEM-modelling

- Aeroelastic stability, specifically for non-metal structures

- Aeroservoelasticity

- Noise prediction: high fidelity methods and derived low order models

- Inverse design and optimization through adjoint equations

American Institute of Aeronautics and Astronautics 


\section{Conclusions and Outlook}

The aerospace industry and the wind energy industry share very similar challenges in the decades ahead concerning the following question: How can we reduce the emission of greenhouse gases and so mitigate the effects of climate change? Both fields need to focus on improving the efficiency of their designs: low drag and lightweight constructions. It is in the technical problems associated with these targets that the two industries can collaborate in their research.

For wind turbine aerodynamics and aeroelasticity simulations in engineering design environments one of the solutions points towards strong viscous-inviscid interaction techniques that were developed some decades ago in the aerospace field. The coupling of a panel method for the external flow with a method solving the integral boundary layer equations restricts the flow problem to the rotor blade and its wake surfaces.

The interaction between aerodynamic forces and structural dynamics should be taken into account to predict the behavior of a real wind turbine. Ideally, when also the structure is represented by a surface model, it is possible to account in the panel method approach for the effects of local deformations and buckling of the rotor blade. Together with accurate laminar-turbulent transition prediction methods such techniques could be applied in wind turbine engineering environments with short problem turnaround times at small scale computer systems. Using a combination of panel method and integral boundary layer method, such fluid-structure interaction approach is much less cumbersome than an approach based on solving the Navier-Stokes equations using a volumetric discretization of the 3D computational domain.

\section{References}

1. A. Betz and L. Prandtl, Schraubenpropeller mit Geringstem Energieverlust, Nachrichten von der Gesellschaft der Wissenschaften zu Göttingen, 1919.

2. A. Betz, Wind Energie und ihre Ausnutzung durch Windmühlen, Vandenhoeck \& Ruprecht, 1926.

3. O. de Vries, Fluid Dynamic Aspects of Wind Energy Conversion, AGARD-AG-243. AGARD, 1978.

4. J.N. Sørensen, General Momentum Theory for Horizontal Axis Wind Turbines, Springer, 2016.

5. G.A.M van Kuik, J.N. Sørensen, and V.L. Okulov, Rotor theories by Professor Joukowsky: Momentum theories, Progress in Aerospace Sciences, 73:1-18, 2015.

6. V.L. Okulov, J.N. Sørensen, and D.H. Wood. The rotor theories by Professor Joukowsky: Vortex theories, Progress in Aerospace Sciences, 73:19-46, 2015.

7. D.A. Grier. When Computers Were Human, Princeton University Press, Princeton, USA, 2005.

8. E.T.G. Bot, FarmFlow validation against full scale wind farms, ECN report ECN-E--15-045, 2015.

9. H. Özdemir, M.C. Versteeg, A.J. Brand, Improvements in ECN Wake Model, ICOWES2013 Conference, 2013.

10. B. Sanderse, S.P. van der Pijl, and B. Koren, Review of computational fluid dynamics for wind turbine wake aerodynamics, Wind Energy 14, 799, 2011.

11. S. ten Pas, CFD simulations of the MEXICO wind turbine. Validating ENSOLV for wind turbine flows. MSc thesis, University of Twente, 2016.

12. K. Boorsma and J.G. Schepers, New MEXICO experiment. Preliminary overview with initial validation, ECN-E--14-048, Energy research Centre of the Netherlands, 2014.

13. M.M. Hand, D.A. Simms, L.J. Fingersh, D.W. Jager, J.R. Cotrell, S. Schreck, and S.M. Larwood, Unsteady Aerodynamics Experiment Phase VI Wind Tunnel Test Configurations and Available Data Campaigns, NREL/TP-500-29955, National Renewable Energy Laboratory, NREL, 2001.

14. C.P. van Dam, E.A. Mayda, D.D. Chao, D.E. Berg, Computational Design and Analysis of Flatback Airfoil Wind Tunnel Experiment, SANDIA Report, SAND2008-1782, 2008.

15. P.S. Veers, Three-Dimensional Wind Simulation, SAND88-0152, Sandia National Laboratories, 1988.

16. D. Winkelaar, Fast three dimensional wind simulation and the prediction of stochastic blade loads, 10'th ASME Wind Energy Symposium, Houston, 1991.

17. N.D. Kelley, Full Vector (3-D) Inflow Simulation in Natural and Wind Farm Environments Using an Expanded Version of the SNLWIND (Veers) Turbulence Code, NREL/TP-442-5225, National Renewable Energy Laboratory, NREL, 1992.

18. J. Mann, Wind field simulation, Probabilistic Engineering Mechanics, Volume 13, Issue 4, Pages 269-282, 1998.

19. M-C. Hsu, Y. Bazilevs, Fluid-structure interaction modeling of wind turbines: simulating the full machine, Computational Mechanics, Volume 50, Issue 6, pp 821-833, 2012.

20. A. van Garrel, Multilevel Panel Method for Wind Turbine Rotor Flow Simulations, PhD thesis, University of Twente, 2016.

21. A. van Garrel, C.H. Venner, and H.W.M. Hoeijmakers, Fast Multilevel Panel Method for Wind Turbine Rotor Flow Simulations, 35th Wind Energy Symposium, AIAA SciTech Forum, AIAA 2017-2001, 2017.

22. E.A. Prieto Serratos, Multilevel Panel Method Validation Using the New MEXICO Wind Tunnel Measurements, MSc thesis, University of Twente, 2017.

American Institute of Aeronautics and Astronautics 
23. R.C. Lock and B.R. Williams, Viscous-Inviscid Interactions in External Aerodynamics, Progress in Aerospace Sciences, 24:51-171, 1987.

24. M. Drela, XFOIL: An Analysis and Design System for Low Reynolds Number Airfoils, In T.J. Mueller, editor, Low Reynolds Number Aerodynamics, Vol. 54 of Lecture Notes in Engineering, pages 1-12. Springer-Verlag, New York, 1989.

25. M. Drela. XFOIL. http://web.mit.edu/drela/Public/web/xfoil/.

26. H. Snel, R. Houwink, and J. Bosschers, Sectional Prediction of Lift Coefficients on Rotating Wind Turbine Blades in Stall, ECN-C--93-052, Energy research Centre of the Netherlands, 1994.

27. R.P.J.O.M. van Rooij, Modifications of the boundary layer calculation in RFOIL for improved airfoil stall prediction, Delft University of Technology, Report IW-96087R, 1996.

28. G. Ramanujam, H. Özdemir, and H.W.M. Hoeijmakers, Improving Airfoil Drag Prediction, Journal of Aircraft, Vol. 53, No. 6, pp. 1844-1852, 2016.

29. G. Ramanujam and H. Özdemir, Improving Airfoil Lift Prediction, 35th Wind Energy Symposium, AIAA SciTech Forum, AIAA 2017-1999, 2017.

30. R. Vaithiyanathasamy, H. Özdemir, G. Bedon, A. van Garrel, A double wake model for interacting boundary layer methods, accepted to 36th Wind Energy Symposium, AIAA SciTech Forum, 8-12 January 2018, Florida, USA.

31. N. Ramos-García, Unsteady Viscous-Inviscid Interaction Technique for Wind Turbine Airfoils, PhD thesis, Technical University of Denmark, 2011.

32. N. Ramos-García, J.N. Sørensen, and W.Z. Shen, Three-dimensional viscous-inviscid coupling method for wind turbine computations, Wind Energy, 19(1):67-93, 2016.

33. A.J. van der Wees and J. van Muijden, A Robust Quasi-Simultaneous Interaction Method for a Full Potential Flow with a Boundary Layer with Application to Wing-Body Configurations, In Fifth Symposium on Numerical and Physical Aspects of Aerodynamic Flows, 1992.

34. A.E.P. Veldman, New, Quasi-Simultaneous Method to Calculate Interacting Boundary Layers, AIAA Journal, 19(1):79-86, 1981.

35. A. van Garrel, Integral boundary layer methods for wind turbine aerodynamics, Report ECN-C--04-004, Energy research Centre of the Netherlands, 2004.

36. A. van Garrel, Development of a Wind Turbine Rotor Flow Panel Method, Report ECN-E--11-071, Energy research Centre of the Netherlands, 2011.

37. H. Özdemir, Development of a discontinuous Galerkin method for the unsteady integral boundary layer equations, 8th Euromech Fluid Mechanics Conference, Bad Reichenhall, Germany, 13-16 September 2010.

38. H. Özdemir and E.F. van den Boogaard, Solving the integral boundary layer equations with a discontinuous Galerkin method, EWEA 2011 Scientific Proceeding, pp.229-233, 2011.

39. H.A. Bijleveld, A Quasi-Simultaneous Interaction Method for the Determination of Aerodynamic Forces on Wind Turbine Blades, PhD thesis, University of Groningen, 2013.

40. H. Özdemir, A. van Garrel, A. Koodly Ravishankara, F. Passalacqua, and H. Seubers, Unsteady Interacting Boundary Layer Method, 35th Wind Energy Symposium, AIAA SciTech Forum, AIAA 2017-2003, 2017.

41. M. Drela, Three-Dimensional Integral Boundary Layer Formulation for General Configurations, AIAA Paper 2013-2437, AIAA, 2013.

42. S. Zhang, M.C. Galbraith, S.R. Allmaras, M. Drela, D.L. Darmofal, A Non-parametric Discontinuous Galerkin Formulation of the Integral Boundary Layer Equations with Strong Viscous/Inviscid Coupling, 23rd AIAA Computational Fluid Dynamics Conference, AIAA AVIATION Forum, AIAA 2017-4278, 2017.

43. B. Oskam, Transonic Panel Method for the Full Potential Equation Applied to Multicomponent Airfoils, AIAA Journal, 23(9):1327-1334, 1985.

44. A. Rottgermann, Eine Methode zur Berucksichtigung kompressibler und transsonischer Effekte in Randelementverfahren, PhD thesis, University of Stuttgart, 1994.

45. A. Brandt and A.A. Lubrecht, Multilevel Matrix Multiplications and Fast Solution of Integral Equations, Journal of Computational Physics, 90:348-370, 1990.

American Institute of Aeronautics and Astronautics 